\title{
$\mathrm{KJCCM}$
}

\section{Acute Physiology and Chronic Health Evaluation II Score and Sequential Organ Failure Assessment Score as Predictors for Severe Trauma Patients in the Intensive Care Unit}

\author{
Min A Lee, Kang Kook Choi, Byungchul Yu, Jae Jeong Park, Youngeun Park, Jihun Gwak, \\ Jungnam Lee, Yang Bin Jeon, Dae Sung Ma, and Gil Jae Lee \\ Department of Trauma, Gachon University Gil Medical Center, Incheon, Korea
}

\begin{abstract}
Background: The Acute Physiology and Chronic Health Evaluation (APACHE) II scoring system and the Sequential Organ Failure Assessment (SOFA) scoring system are widely used for critically ill patients. We evaluated whether APACHE II score and SOFA score predict the outcome for trauma patients in the intensive care unit (ICU).

Methods: We retrospectively analyzed trauma patients admitted to the ICU in a single trauma center between January 2014 and December 2015. The APACHE II score was figured out based on the data acquired from the first 24 hours of admission; the SOFA score was evaluated based on the first 3 days in the ICU. A total of 241 patients were available for analysis. Injury Severity score, APACHE II score, and SOFA score were evaluated.

Results: The overall survival rate was $83.4 \%$. The non-survival group had a significantly high APACHE II score $(24.1 \pm 8.1$ vs. $12.3 \pm 7.2$ $\mathrm{P}<0.001)$ and SOFA score $(7.7 \pm 1.7$ vs. $4.3 \pm 1.9, \mathrm{P}<0.001)$ at admission. SOFA score had the highest areas under the curve (0.904). During the first 3 days, SOFA score remained high in the non-survival group. In the non-survival group, cardiovascular system, neurological system, renal system, and coagulation system scores were significantly higher.

Conclusions: In ICU trauma patients, both SOFA and APACHE II scores were good predictors of outcome, with the SOFA score being the most effective. In trauma ICU patients, the trauma scoring system should be complemented, recognizing that multi-organ failure is an important factor for mortality.
\end{abstract}

Key Words: APACHE II; intensive care units; mortality; multiple trauma; Sequential Organ Failure Assessment.

\section{Introduction}

Objective assessment of the severity of trauma is necessary for patient triage, outcome comparison, and quality assessment. The trauma scoring system was originally developed to categorize patients on scene. There are several scoring systems, such as Abbreviated Injury scale (AIS), Injury Severity score (ISS), Glasgow Coma scale (GCS), Revised Trauma score (RTS), and Trauma and Injury Severity score (TRISS).

Multiple trauma patients usually visit the intensive care unit (ICU) after resuscitation, emergency operation, or intervention. Postinjury multiple-organ failure remains the most important cause of late trauma deaths, leading to a signifi-

Received on April 26, 2017 Revised on June 1, 2017 Accepted on June 2, 2017

Correspondence to: Gil Jae Lee, Department of Trauma, Gachon University Gil Medical Center, 21 Namdong-daero 774beon-gil, Namdong-gu, Incheon 21565, Korea Tel: +82-32-460-2140, Fax: +82-32-461-2371, E-mail: nonajugi@gilhospital.com

cc This is an Open Access article distributed under the terms of the Creative Commons Attribution Non-Commercial License (http://creativecommons.org/ licenses/by-nc/4.0/) which permits unrestricted non-commercial use, distribution, and reproduction in any medium, provided the original work is properly cited. Copyright (C) 2017 The Korean Society of Critical Care Medicine 
cant use of intensive care resources [1]. To date, prospective analyses that compare standard trauma scores and scores designated for the critically ill are missing. Studies on ICU scoring generally contain multiple-trauma patients only as subgroups [2,3].

The Acute Physiology and Chronic Health Evaluation II (APACHE II) score uses a point-score system based on patient's age, physiologic measurement, and medical history. This system is able to classify a variety of patients depending on the prognosis due to the intensive relationship between acute physiologic dysfunction and the risk of death due to illness [4].

At first, The Sequential Organ Failure Assessment (SOFA) score was used to describe critically ill patients in the ICU. It is not used as a categorical cutoff point to clarify multi-organ failure; rather, it is used as a ceaseless descriptive variable for continuous monitoring [5]. An increase in SOFA score during the early ICU period predicts a high mortality rate [6].

These models were designed for critically ill patients. We aimed to analyze the effectiveness of these indicators in predicting the outcome for patients who visited the ICU due to severe trauma compared to the trauma scoring system.

\section{Materials and Methods}

We retrospectively analyzed trauma patients admitted to the ICU of a single trauma center between January 2014 and December 2015. The inclusion criteria were as follows: patients who had at least 15 ISS points were 18 years of age or older, and were in the ICU for more than 72 hours. The non-mechanical trauma mechanism (burns, hanging, electrocution, poisoning, or drowning) and missing data were excluded. Patients with major head injury (AIS of head $\geq 4$ ) were also excluded, owing to different physiologic responses to severe trauma and a significantly different mortality and morbidity profile [7]. Retrospective chart review was performed, and ISS, APACHE II score, and SOFA score were evaluated using patient data. These scores were compared between two groups: the survivors group and the non-survivors group.

ISS and TRISS were obtained from emergency room admission data. The APACHE II score was calculated based on the data obtained from the first 24 hours of ICU admission. The SOFA score was evaluated based on the first 3 days in the ICU. APACHE II uses a point score based on the initial values of 12 routine physiologic measurements (body temperature, mean arterial blood pressure, heart rate, respiratory rate, oxygenation, arterial $\mathrm{PH}$, hematocrit, white blood cell count, serum levels of sodium, potassium, creatinine, and GCS), age, as well as previous health status to provide a general measure of disease severity [4]. We used the worst value for each variable. SOFA is composed of scores from six organ systems (respiratory, cardiovascular, hepatic, coagulation, renal, and neurological), graded from 0 to 4 by degree of dysfunction [6]. We calculated the SOFA score as described by Vincent et al. [8].

A univariate analysis was performed with the Student t-test for continuous variables or the chi-square test for categorical variables. The receiver's operating characteristic was calculated for each score, and the areas under the curve (AUC) were compared to determine which scores were useful for predicting mortality. Statistical significance was accepted at $\mathrm{P}<0.05$.

\section{Results}

A total of 241 patients were included during the study period. Of these, 241 trauma patients were enrolled; the overall survival rate was $83.4 \%$. Mortality was assessed at 28 days. We divided these patients into two groups: the survival group and the non-survival group. There were no differences in age, sex, or mechanism between the two groups. There was a difference in the number of days with a ventilator (4.4 days vs. 11.8 days, $\mathrm{P}=0.001$ ), the amount of blood transfused during the initial 4 hours (3.32 units vs. 9.4 units, $\mathrm{P}<0.001)$, and the amount of transfusion for 24 hours $(2.45$ units vs. 13.6 units, $\mathrm{P}<0.001)$ in the sub- 
Table 1. Demographic characteristics of survivors and non-survivors

\begin{tabular}{lccc}
\hline Characteristic & Survivor $(\mathrm{n}=201)$ & Non-survivor $(\mathrm{n}=40)$ & P-value \\
\hline Age (yr) & 51.4 & 54.7 & 0.246 \\
Male sex (\%) & 70 & 75 & 0.578 \\
ICU stay (d) & 10.4 & 11.8 & 0.188 \\
Ventilator day & 4.4 & 9.4 & 0.001 \\
Transfusion in the first 4 hr (unit of RBCs) & 3.32 & 13.6 & $<0.001$ \\
Transfusion in the first 24 hr (unit of RBCs) & 2.45 & 92.5 & $<0.001$ \\
Mechanism (blunt, \%) & 98 & 0.058 \\
\hline
\end{tabular}

ICU: intensive care unit; $\mathrm{RBC}$ : red blood cell.

Table 2. Mean scores for TRISS, APACHE II, SOFA, ISS, and RTS

\begin{tabular}{lccc}
\hline Variable & Survivor $(\mathrm{n}=\mathbf{2 0 1})$ & Non-survivor $(\mathrm{n}=40)$ & P-value \\
\hline TRISS & $83.6 \pm 21.9$ & $49.5 \pm 31.2$ & $<0.001$ \\
APACHE $\|$ & $12.3 \pm 7.2$ & $24.1 \pm 8.0$ & $<0.001$ \\
SOFA (mean) & $4.3 \pm 1.9$ & $7.7 \pm 1.7$ & $<0.001$ \\
ISS & $24.7 \pm 8.2$ & $29.5 \pm 12.3$ & 0.002 \\
RTS & $6.92 \pm 1.4$ & $4.32 \pm 2.5$ & $<0.001$ \\
\hline
\end{tabular}

Values are presented as mean \pm standard deviation.

TRISS: Trauma and Injury Severity score; APACHE: Acute Physiology and Chronic Health Evaluation; SOFA: Sequential Organ Failure Assessment; ISS: Injury Severity score; RTS: Revised Trauma score.

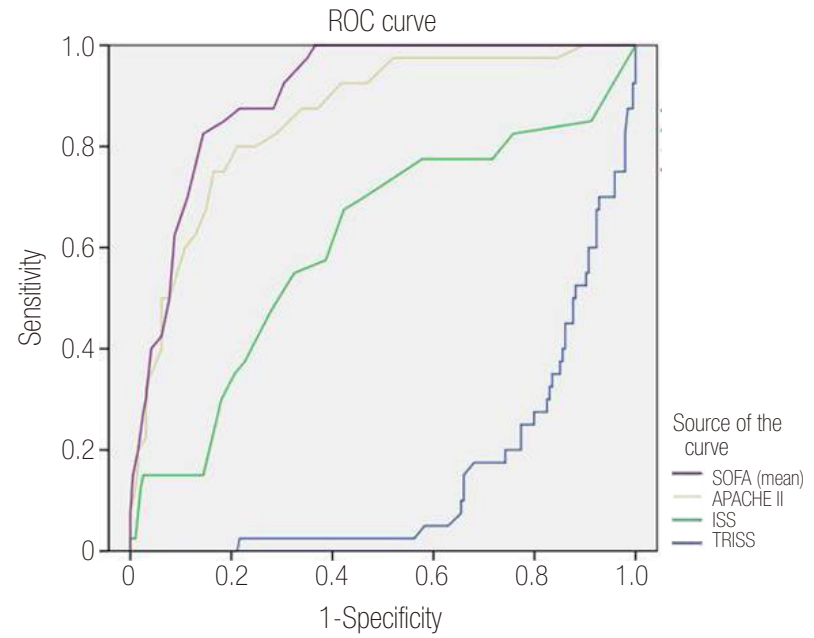

Figure 1. Receiver operating characteristic (ROC) curves for APACHE II, SOFA (mean), TRISS, and ISS. SOFA: Sequential Organ Failure Assessment; APACHE: Acute Physiology and Chronic Health Evaluation; ISS: Injury Severity score; TRISS: Trauma and Injury Severity score.

\section{groups (Table 1).}

Comparing the scores between the two groups, the nonsurvival group showed higher scores on both APACHE
II (24.1 vs. $12.3, \mathrm{P}<0.001)$ and SOFA (7.7 vs. $4.3, \mathrm{P}<$ $0.001)$. Moreover, there were significant differences in TRISS (49.5 vs. 83.6, $\mathrm{P}<0.001)$, ISS (29.5 vs. 24.7, $\mathrm{P}=$ 0.002 ), and RTS (4.32 vs. 6.92, $\mathrm{P}<0.001$ ) (Table 2).

The AUC of the receiver operating characteristic was calculated to compare the capability of each scoring system to predict death. The SOFA score, APACHE II score, TRISS, and ISS were 0.904, 0.859, 0.147, and 0.613, respectively. The SOFA score was the highest at $0.904(\mathrm{P}<$ 0.001) (Figure 1). Using the cutoff point, the sensitivity and specificity of each scoring system were compared. The scores for SOFA sensitivity and specificity were $83 \%$ and $86 \%$, respectively, which were superior compared to the other scoring systems (Table 3 ).

During the first 3 days, SOFA score remained high in the non-survival group (day $1,3.85$ vs. $6.75, \mathrm{P}<0.001$; day $2,4.54$ vs. $8.07, \mathrm{P}<0.001$; day $3,4.43$ vs. $8.45, \mathrm{P}<$ $0.001)$. Cardiovascular system (0.57 vs. $2.95, \mathrm{P}<0.001)$, neuromuscular system (1.54 vs. 3.60, $\mathrm{P}<0.001)$, renal system $(0.08$ vs. $0.40, \mathrm{P}<0.001)$, coagulation system 
Table 3. Comparison of scoring system predictive values

\begin{tabular}{lccccc}
\hline Variable & Cutoff point & P-value & Sensitivity (\%) & Specificity (\%) & AUC \\
\hline TRISS & 78.5 & $<0.001$ & 25 & 21 & 0.147 \\
ISS & 25.5 & 0.024 & 58 & 61 & 0.613 \\
APACHE II & 54.7 & $<0.001$ & 80 & 79 & 0.859 \\
SOFA & 6.5 & $<0.001$ & 83 & 86 & 0.904 \\
\hline
\end{tabular}

AUC: areas under the curve; TRISS: Trauma and Injury Severity score; ISS: Injury Severity score; APACHE: Acute Physiology and Chronic Health Evaluation; SOFA: Sequential Organ Failure Assessment.

Table 4. Severity value of SOFA scores for each organ during the first 3 days

\begin{tabular}{|c|c|c|c|c|}
\hline Day & SOFA score & Survivor & Non-survivor & P-value \\
\hline \multirow[t]{7}{*}{ Day 1} & SOFA score & 3.85 & 6.75 & $<0.001$ \\
\hline & Respiratory system & 0.43 & 0.40 & 0.509 \\
\hline & Coagulation & 0.67 & 1.00 & 0.037 \\
\hline & Liver & 0.19 & 0.35 & 0.117 \\
\hline & Cardiovascular system & 0.57 & 2.95 & $<0.001$ \\
\hline & Neurological system & 1.54 & 3.60 & $<0.001$ \\
\hline & Renal system & 0.08 & 0.40 & $<0.001$ \\
\hline \multirow[t]{7}{*}{ Day 2} & SOFA score & 4.54 & 8.07 & $<0.001$ \\
\hline & Respiratory system & 0.43 & 0.57 & 0.299 \\
\hline & Coagulation & 1.27 & 1.75 & 0.007 \\
\hline & Liver & 0.35 & 0.43 & 0.618 \\
\hline & Cardiovascular system & 0.37 & 2.73 & $<0.001$ \\
\hline & Neurological system & 1.35 & 3.70 & $<0.001$ \\
\hline & Renal system & 0.10 & 0.63 & $<0.001$ \\
\hline \multirow[t]{7}{*}{ Day 3} & SOFA score & 4.43 & 8.45 & $<0.001$ \\
\hline & Respiratory system & 0.40 & 0.43 & 0.844 \\
\hline & Coagulation & 1.37 & 1.90 & 0.001 \\
\hline & Liver & 0.38 & 0.53 & 0.259 \\
\hline & Cardiovascular system & 0.25 & 2.53 & $<0.001$ \\
\hline & Neurological system & 1.15 & 3.63 & $<0.001$ \\
\hline & Renal system & 0.12 & 0.98 & $<0.001$ \\
\hline
\end{tabular}

SOFA: Sequential Organ Failure Assessment.

(0.67 vs. $1.00, \mathrm{P}=0.037)$ scores were all significantly higher in the non-survival group (Table 4).

\section{Discussion}

Trauma is a significant health problem, as it is a major cause of death in younger adults and adolescents. The mortality of severely injured patients is reported to vary from $7 \%$ to $45 \%$ [9]. The reason for this variance is that there may be a difference in the quality of management between centers and between countries, as well as differences in individual patient characteristics. Trauma scoring systems should provide indices that overcome these differences. We need a system that can accurately quantify injury, evaluate outcomes, and evaluate therapeutic quality $[10,11]$. Over the past 30 years, variant trauma scoring systems have been developed. Most of 
these systems are designed with anatomical or physiological components, and there are some scoring systems that combine the two components. However, there is still no accurate system that has been widely accepted. Physiological disorder as well as anatomical injury also affect post-traumatic mortality. In particular, physiologic disarrangement has a significant impact on late mortality and multi-organ failure. Therefore, the addendum of physiological data to the scoring systems is crucial [9].

In this study, we compared the scores of SOFA, APACHE II, and TRISS; all three scoring systems were significantly able to predict death. The AUC of SOFA was the highest with 0.904, followed by APACHE II (AUC, 0.859), and TRISS was the lowest with 0.147 . The SOFA score was not naturally designed to predict mortality [8]. It was designed to describe the degree of organ failure in critical patients with a high risk of many organ dysfunctions. However, even in patients with critically ill patients or multiple trauma groups, SOFA predicts outcomes well $[3,6,12]$. Antonelli et al. [13] concluded that SOFA score may be helpful to find out multiple trauma patients with poor prognosis and a higher probability of prolonged ICU stay. Hwang et al. [14] reported that the SOFA score was helpful in forecasting the result of ICU trauma patients. And, SOFA scoring system is simple and can easily be compared with other scoring systems.

In our study, we excluded severe head trauma (AIS of head $\geq 4$ ). But, neuromuscular system scores were significantly higher in the non-survival group during the first 3 days. The non-survival group had more shock state patients, traumatic brain injury (AIS $\leq 3$ ) patients, and diffuse axonal injury patients who were not confirmed by magnetic resonance imaging and were missing from AIS coding.

APACHE II has been shown to be a significant predictor of outcomes for ICU trauma patients. Hwang et al. [14] reported that APACHE II scores were practical for predicting the outcomes of ICU trauma patients $(52.4 \%$ sensitivity, $94.8 \%$ specificity, and $86 \%$ accuracy). In addition, Wong et al. [15] said that APACHE II scores accurately predicted mortality in ICU trauma patients.
APACHEII has several advantages over TRISS. Physiologic components have 12 in APACHEII, whereas TRISSI has three. It has been demonstrated that physiologic measurement has a significant impact on the result of ICU patients [16]. It also includes underlying diseases. This can improve the predictive power for ICU trauma patients. Milzman et al. [17] concluded that preinjury illness had a significantly disadvantage on survival of trauma patients.

The APACHE II score was developed for critically ill patients with different clinical features from trauma patients. Because APACHE II considers only patient status in the ICU, the possibility of death may be underestimated if the patient is stabilized through resuscitation in the emergency room [9]. Additionally, anatomical components are absent in the APACHE II system. McAnena et al. [18] reported that although APACHE II may well determine the acute status of patients with chronic medical disease, it is necessary to complement anatomical components. However, this study included patients who died within the first 24 hours of visiting the emergency room. The APACHE II system is also effective in trauma patients in the ICU, except for early death, as shown in this study.

TRISS unites RTS, ISS, patient's age, and the type of injury [19]. It has been widely used to assess traumas and to predict outcomes [15]. However, it does not include an assessment of chronic health status. TRISS also contains only three physiological factors [9].

In this study, TRISS had the lowest predictive power for mortality. This is probably because the study groups were included for more than 3 days, except for cases with early death due to trauma. Initial physiologic state and injury severity are also important in this group; however, multi-organ failure is more influential for mortality in late deaths. Multi-organ failure is still considered the main cause of late post-injury mortality [20]. Ciesla et al. [7] reported the proportion of multi-organ failure in highrisk trauma patients was $25 \%$. Moreover, posttraumatic multi-organ failure caused approximately $51 \%$ of late trauma deaths. Norris et al. [21] concluded that TRISS 
should progress for more meaningful interpretation.

There are several limitations to consider when interpreting our results. Our study was limited to a cohort from a single center. The study period was relatively short, only 2 years. The number of patients was not large, and we included only patients who were admitted to the ICU. We excluded patients who died within 72 hours after ICU admission. Excluding early death was done to compare APACHE II, SOFA, and TRISS for measuring late death in ICU. Follow-up study involving early death and late death will be needed in the future. And largescale prospective studies should be performed to better determine more accurate and complementary predictors. In conclusion, in ICU trauma patients, both SOFA and APACHE II scores were good predictors of outcome. SOFA was the most effective among them. In ICU trauma patients, predictors should complement the trauma scoring system, with recognition that multi-organ failure is an important factor in mortality.

\section{ORCID}

Min A Lee

http://orcid.org/0000-0002-9725-8993

\section{References}

1. Evans JA, van Wessem KJ, McDougall D, Lee KA, Lyons T, Balogh ZJ. Epidemiology of traumatic deaths: comprehensive population-based assessment. World J Surg 2010;34:158-63.

2. Lefering R, Goris RJ, van Nieuwenhoven EJ, Neugebauer E. Revision of the multiple organ failure score. Langenbecks Arch Surg 2002;387:14-20.

3. Moreno R, Vincent JL, Matos R, Mendonça A, Cantraine F, Thijs L, et al. The use of maximum SOFA score to quantify organ dysfunction/failure in intensive care: results of a prospective, multicentre study. Working group on sepsis related problems of the ESICM. Intensive Care Med 1999;25:686-96.
4. Knaus WA, Draper EA, Wagner DP, Zimmerman JE. APACHE II: a severity of disease classification system. Crit Care Med 1985;13:818-29.

5. Dewar DC, White A, Attia J, Tarrant SM, King KL, Balogh ZJ. Comparison of postinjury multiple-organ failure scoring systems: Denver versus Sequential Organ Failure Assessment. J Trauma Acute Care Surg 2014;77:624-9.

6. Ferreira FL, Bota DP, Bross A, Mélot C, Vincent JL. Serial evaluation of the SOFA score to predict outcome in critically ill patients. JAMA 2001;286:1754-8.

7. Ciesla DJ, Moore EE, Johnson JL, Burch JM, Cothren CC, Sauaia A. A 12-year prospective study of postinjury multiple organ failure: has anything changed? Arch Surg 2005;140:432-8.

8. Vincent JL, Moreno R, Takala J, Willatts S, De Mendonça A, Bruining H, et al. The SOFA (Sepsis-related Organ Failure Assessment) score to describe organ dysfunction/failure. On behalf of the working group on sepsis-related problems of the European Society of Intensive Care Medicine. Intensive Care Med 1996;22:707-10.

9. Chawda MN, Hildebrand F, Pape HC, Giannoudis PV. Predicting outcome after multiple trauma: which scoring system? Injury 2004;35:347-58.

10. Baker SP, O’Neill B. The Injury Severity score: an update. J Trauma 1976;16:882-5.

11. Champion HR, Copes WS, Sacco WJ, Frey CF, Holcroft JW, Hoyt DB, et al. Improved predictions from a severity characterization of trauma (ASCOT) over Trauma and Injury Severity score (TRISS): results of an independent evaluation. J Trauma 1996;40:42-8.

12. Kajdacsy-Balla Amaral AC, Andrade FM, Moreno R, Artigas A, Cantraine F, Vincent JL. Use of the sequential organ failure assessment score as a severity score. Intensive Care Med 2005;31:243-9.

13. Antonelli M, Moreno R, Vincent JL, Sprung CL, Mendoça A, Passariello M, et al. Application of SOFA score to trauma patients: Sequential Organ Failure Assessment. Intensive Care Med 1999;25:389-94. 
14. Hwang SY, Lee JH, Lee YH, Hong CK, Sung AJ, Choi YC. Comparison of the Sequential Organ Failure Assessment, Acute Physiology and Chronic Health Evaluation II scoring system, and Trauma and Injury Severity score method for predicting the outcomes of intensive care unit trauma patients. Am J Emerg Med 2012;30:749-53.

15. Wong DT, Barrow PM, Gomez M, McGuire GP. A comparison of the Acute Physiology and Chronic Health Evaluation (APACHE) II score and the Trauma-Injury Severity score (TRISS) for outcome assessment in intensive care unit trauma patients. Crit Care Med 1996;24:1642-8.

16. Knaus WA. Why measure severity? Reanim Urgences 1994;3:159-63.

17. Milzman DP, Boulanger BR, Rodriguez A, Soder- strom CA, Mitchell KA, Magnant CM. Pre-existing disease in trauma patients: a predictor of fate independent of age and injury severity score. J Trauma 1992;32:236-43.

18. McAnena OJ, Moore FA, Moore EE, Mattox KL, Marx JA, Pepe P. Invalidation of the APACHE II scoring system for patients with acute trauma. J Trauma 1992;33:504-6.

19. Boyd CR, Tolson MA, Copes WS. Evaluating trauma care: the TRISS method--Trauma Score and the Injury Severity score. J Trauma 1987;27:370-8.

20. Dewar D, Moore FA, Moore EE, Balogh Z. Postinjury multiple organ failure. Injury 2009;40:912-8.

21. Norris R, Woods R, Harbrecht B, Fabian T, Rhodes M, Morris J, et al. TRISS unexpected survivors: an outdated standard? J Trauma 2002;52:229-34. 